\title{
Coulomb Interaction Effects on Pion Production in Au+Au Collisions at Relativistic Energies
}

\author{
Catalin Ristea $\mathbb{D},{ }^{1,2}$ Oana Ristea $\mathbb{D}^{1},{ }^{1}$ and Alexandru Jipa $\mathbb{D}^{1}$ \\ ${ }^{1}$ Faculty of Physics, University of Bucharest, Magurele, Bucharest, Romania \\ ${ }^{2}$ Institute of Space Science, Magurele, Bucharest, Romania \\ Correspondence should be addressed to Oana Ristea; oana@brahms.fizica.unibuc.ro
}

Received 7 November 2017; Accepted 1 April 2018; Published 9 May 2018

Academic Editor: Piero Nicolini

Copyright (C) 2018 Catalin Ristea et al. This is an open access article distributed under the Creative Commons Attribution License, which permits unrestricted use, distribution, and reproduction in any medium, provided the original work is properly cited. The publication of this article was funded by SCOAP ${ }^{3}$.

\begin{abstract}
Coulomb effects on charged pion transverse momentum spectra measured in Au+Au collisions at RHIC-BES energies are investigated. From these spectra the $\pi^{-} / \pi^{+}$ratios as a function of transverse momentum are obtained and used to extract the "Coulomb kick", $p_{c}$ (a momentum change due to the Coulomb interaction), and initial pion ratio for three different collision energies and various centrality classes. The Coulomb kick shows a decrease with the increase of beam energy and a clear centrality dependence, with larger values for the most central collisions. The results are connected with the kinetic freeze-out dynamics.
\end{abstract}

\section{Introduction}

One of the main goals of studying heavy-ion collisions at relativistic energies is to characterize the nuclear matter at extreme energy densities and temperatures. The measurements of hadron production and transverse momentum distributions provide information about the complex dynamics of the collision and the different phases through which the collision evolves. There are two freeze-out stages in the system evolution: chemical freeze-out and thermal (kinetic) freezeout. When the inelastic interactions among the particles cease and the particle yields become fixed, the chemical freezeout occurs. After chemical freeze-out, the system continues to expand until the kinetic freeze-out stage, when the elastic interactions stop, and the produced hadrons no longer interact with each other. Before freeze-out, the dynamics of the system is dominated by strong interactions while the longrange Coulomb interactions become important only after kinetic freeze-out.

In heavy-ion collisions at SIS [1, 2], AGS [3-5], and SPS [6-8] energies, the transverse momentum spectra of positively and negatively charged pions show a difference in shape at low $p_{T}$ and these results were interpreted as due to the Coulomb final-state interaction between the charged pions and the positive net-charge of the particle source. The net-charge density depends on the degree of baryon stopping produced in the collision. The collision mechanism is changing with energy from strong baryon stopping at AGS energies to transparent collisions at LHC energies [5, 9-13]. At AGS energies, the colliding nuclei are fully stopped in the collision. The produced charged particles are moving in a Coulomb field produced by the positive net-charge from the stopped charge. The charged pions, as the most abundantly produced and lightest species, are the particles most strongly influenced by the Coulomb field. Therefore, they are accelerated or decelerated and their final (detected) momentum is changed. At higher beam energies (SPS and RHIC energies) the collisions have a larger degree of transparency, in the sense that fewer of the original baryons are found at midrapidity after the collision. Therefore, the net-proton density is reduced and the charged pions are less influenced by the Coulomb field.

The $\mathrm{Au}+\mathrm{Au}$ collisions at different energies in the Beam Energy Scan program (BES) at RHIC are used to explore the QCD phase diagram. By changing the collision energy, one can obtain systems characterized by various temperatures and baryon chemical potentials and different regions on the $T-\mu_{B}$ phase diagram can be investigated. Based on 


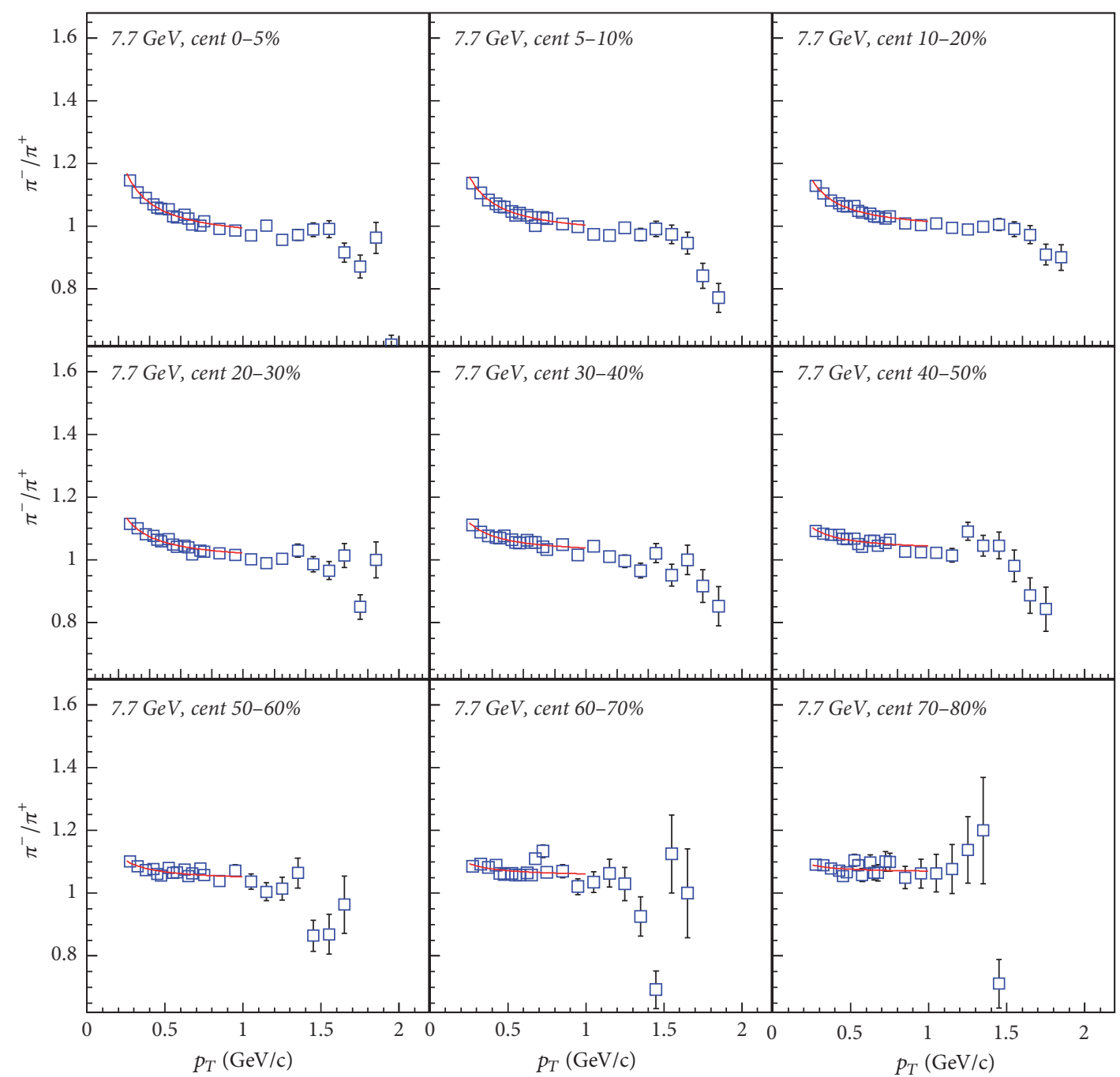

Figure 1: The $\pi^{-} / \pi^{+}$ratios produced in Au+Au collisions at $7.7 \mathrm{GeV}$ as a function of transverse momentum. The data are from [14]. The red lines are the fits with (2).

the BES data, the Coulomb interaction can be studied in a complementary energy domain to AGS and SPS energies.

In this paper, we present an analysis of the Coulomb effects on charged pion production in $\mathrm{Au}+\mathrm{Au}$ collisions at RHIC-BES energies based on an analytic model developed in $[15,16]$. The model considers the longitudinal Bjorken expansion of the fireball and assumes that, on average, a charged pion will receive a momentum change due to the Coulomb interaction or "Coulomb kick", $p_{c}$ :

$$
p_{c} \equiv\left|p_{T}-p_{T, 0}\right| \cong 2 e^{2} \frac{d N^{\mathrm{ch}}}{d y} \frac{1}{R_{f}},
$$

where $p_{T, 0}$ is the transverse momentum at freeze-out, $p_{T}$ is the final transverse momentum, $d N^{\text {ch }} / d y$ is the net-charge distribution, and $R_{f}$ is the kinetic freeze-out radius. The charged pion ratio is

$$
\frac{\pi^{-}}{\pi^{+}}=\left\langle\frac{\pi^{-}}{\pi^{+}}\right\rangle \frac{p_{T}+p_{c}}{p_{T}-p_{c}} \exp \left(\frac{m_{T}^{-}-m_{T}^{+}}{T}\right),
$$

where $m_{T}^{ \pm}=\sqrt{m^{2}+\left(p_{T} \pm p_{c}\right)^{2}},\left\langle\pi^{-} / \pi^{+}\right\rangle$is the initial pion ratio, and $T$ is the kinetic freeze-out temperature (the temperature at the moment when the hadrons decouple from the system).

\section{Results}

In order to extract information related to the Coulomb interaction between the produced particles we analyzed the charged pion transverse momentum spectra produced in $\mathrm{Au}+\mathrm{Au}$ collisions at $\sqrt{s_{\mathrm{NN}}}=7.7,11.5$, and $19.6 \mathrm{GeV}$ and measured by the STAR experiment [17]. The data are from [14].

Based on the STAR published charged pion transverse momentum spectra we obtained the $\pi^{-} / \pi^{+}$ratios as a function of transverse momentum for the collision centrality classes of $0-5 \%, 5-10 \%, 10-20 \%, 20-30 \%, 30-40 \%, 40-50 \%$, $50-60 \%, 60-70 \%$, and $70-80 \%$. The negative to positive pion ratios are shown in Figures 1-3. 


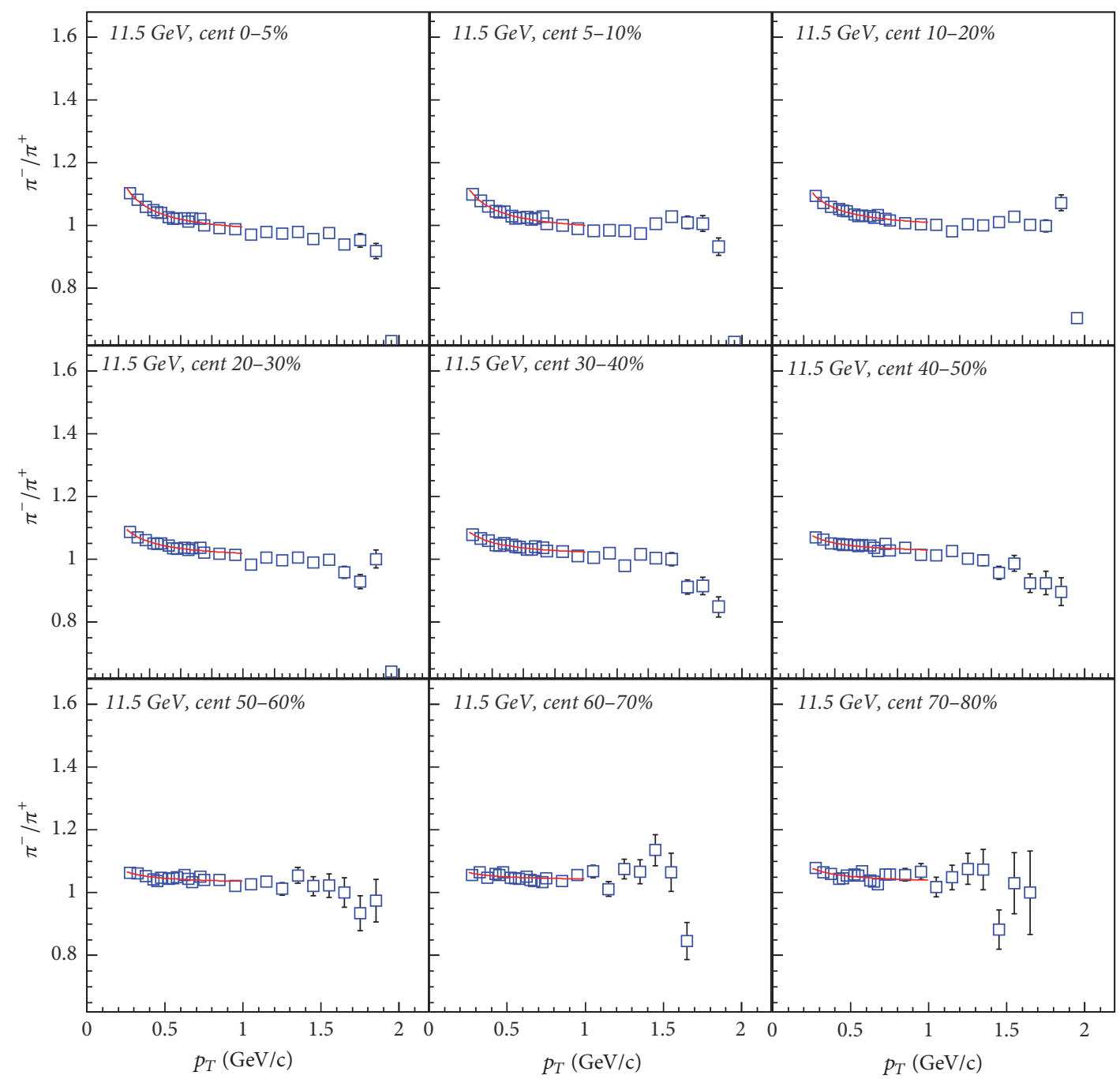

FIgURE 2: The $\pi^{-} / \pi^{+}$ratios produced in $\mathrm{Au}+\mathrm{Au}$ collisions at $11.5 \mathrm{GeV}$ as a function of transverse momentum. The data are from [14]. The red lines are the fits to the ratio data using (2).

The $\pi^{-} / \pi^{+}$ratio is close to unity for all studied energies at higher $p_{T}$. However, at low $p_{T}$ values an increase of the pion ratio above unity is observed for more central collisions. As the beam energy increases, there is still an asymmetry between $\pi^{-}$and $\pi^{+}$production as shown by the low- $p_{T}$ enhancement in ratios, but these effects are much less significant.

The pion ratios were fit with (2). The fit range was chosen $0.25<p_{T}<1 \mathrm{GeV} / \mathrm{c}$, because in the low $p_{T}$ region the Coulomb interaction has a larger influence on the pion spectra and ratios. The two free fit parameters are the Coulomb kick, $p_{c}$, and the initial pion ratio, $\left\langle\pi^{-} \mid \pi^{+}\right\rangle$. The kinetic freeze-out temperatures for each beam energy and collision centrality were taken from [14] and are fixed parameters in this analysis. The fits are shown as solid lines in Figures 1-3 and describe well the charged pion ratios at all energies and collision centralities.

For higher BES beam energies $\left(\sqrt{s_{\mathrm{NN}}}=27 \mathrm{GeV}\right.$ and $39 \mathrm{GeV}$ ), the STAR data show no significant asymmetry between positive and negative pions at low $p_{T}$ and it is not possible to obtain information related to the Coulomb interaction from those data.

To analyze the correlation between the two fit parameters, the contours in the $p_{c}-R_{\pi}$ plane showing 1- $\sigma$ deviation lines from the minimum $\chi^{2}$ values, as well as the values of the fit parameters $p_{c}$ and $R_{\pi}$ (corresponding to the minimum $\chi^{2}$ ) with error bars, are presented in Figure 4. The 1- $\sigma$ uncertainty contours in the $p_{c}-R_{\pi}$ plane were obtained for $\mathrm{Au}+\mathrm{Au}$ collisions at $\sqrt{s_{\mathrm{NN}}}=7.7 \mathrm{GeV}$ (black curves), $\sqrt{s_{\mathrm{NN}}}=$ $11.5 \mathrm{GeV}$ (blue), and $\sqrt{s_{\mathrm{NN}}}=19.6 \mathrm{GeV}$ (red). There is a clear beam energy dependence in the values of the parameters. The contour lines do not overlap for the studied energies; however, in peripheral collisions there is a wider range of possible values compared to more central collisions. As the beam energy increases, the values of $p_{c}$ and $R_{\pi}$ are much more constrained than at $7.7 \mathrm{GeV}$. The $\chi^{2}$ contours indicate that the two parameters are positively correlated; if the Coulomb kick increases, the total pion ratio also increases.

Figure 5 shows the two fit parameters, $p_{c}$ and $R_{\pi}$, as a function of collision centrality expressed as $N_{\text {part }}$ in 


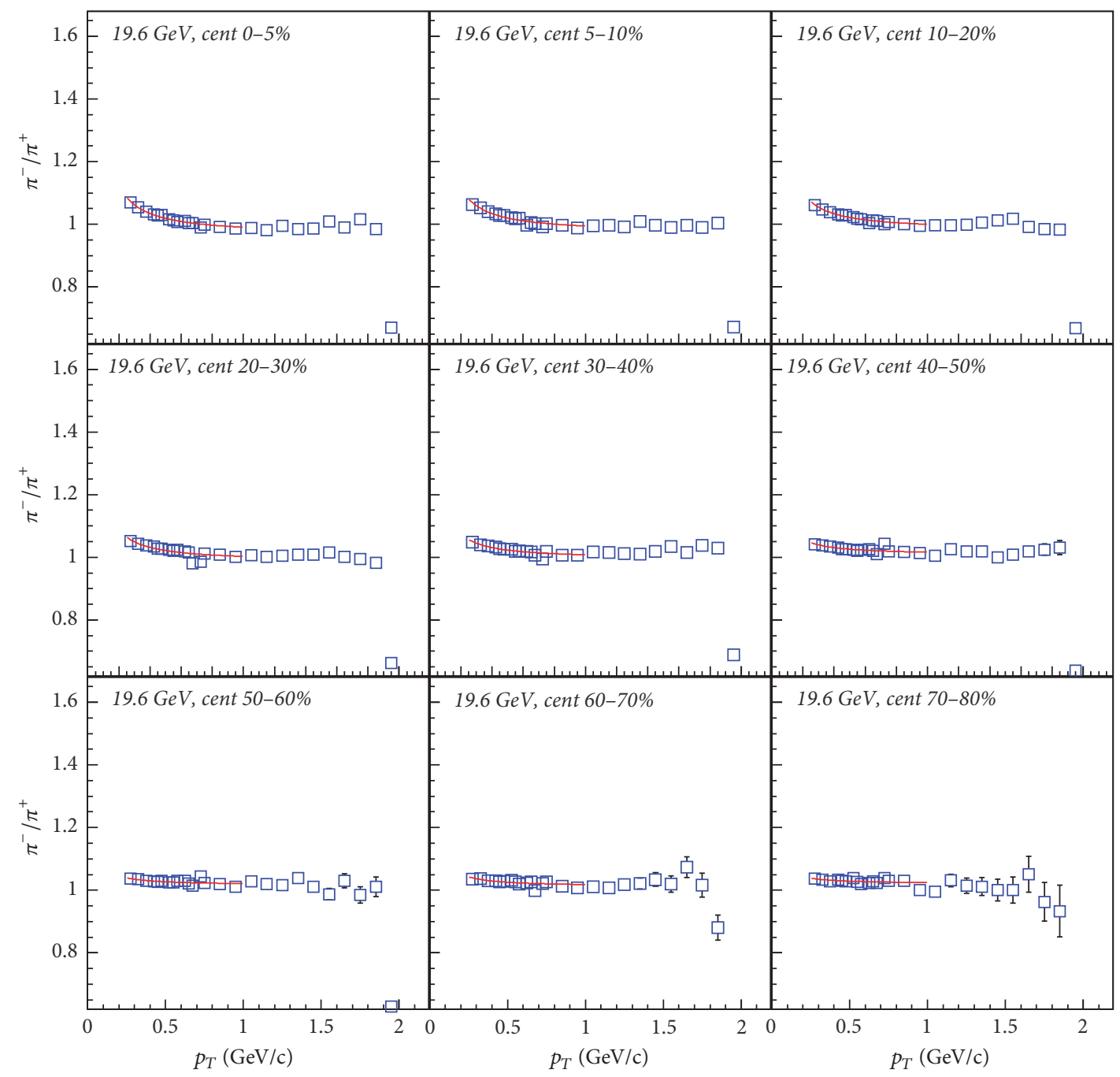

Figure 3: The $\pi^{-} / \pi^{+}$ratios produced in Au+Au collisions at $19.6 \mathrm{GeV}$ as a function of transverse momentum. The data are from [14]. The red lines are the fits to the ratio data using (2).

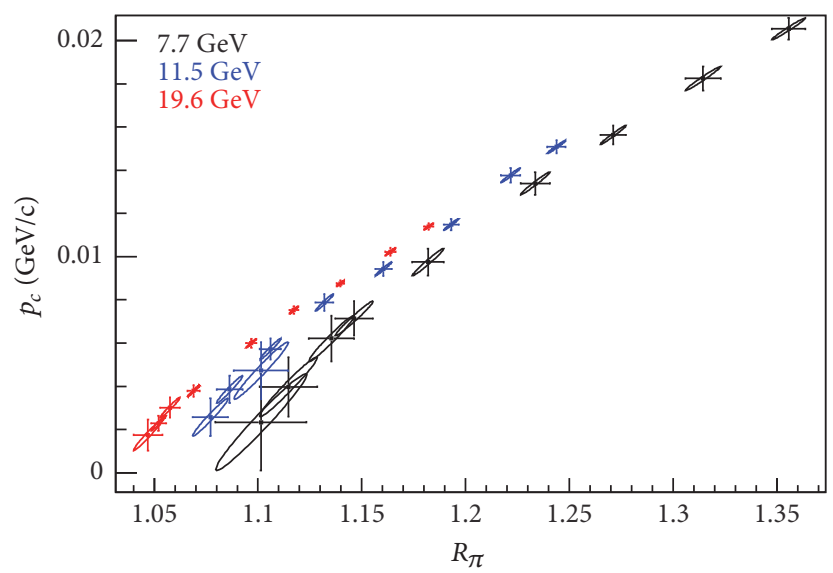

FIGURE 4: Contours in the $p_{c}-R_{\pi}$ plane showing 1- $\sigma$ deviation lines from the minimum $\chi^{2}$ values, as well as the values of the fit parameters $p_{c}$ and $R_{\pi}$ (corresponding to the minimum $\chi^{2}$ ) with error bars. 

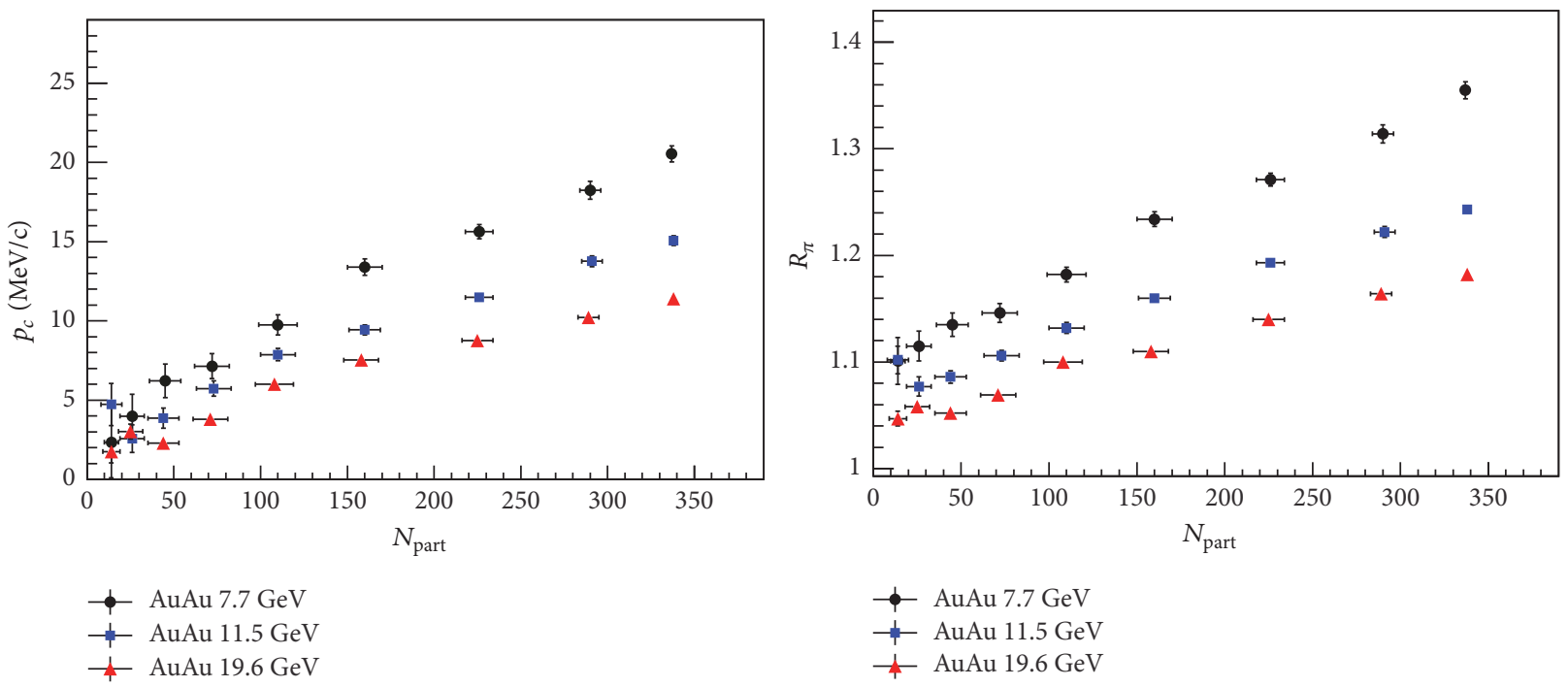

FIGURE 5: The Coulomb kick and initial pion ratio as a function of collision centrality and energy.

$\mathrm{Au}+\mathrm{Au}$ collisions at $7.7 \mathrm{GeV}, 11.5 \mathrm{GeV}$, and $19.6 \mathrm{GeV}$. The centrality dependence of the Coulomb kick is plotted for all energies. The Coulomb kick increases from peripheral to central collisions. The Coulomb kick values are greater at $7.7 \mathrm{GeV}$ for all centralities than at higher BES energies. This may be due to large baryon stopping at midrapidity at the lower energy of $7.7 \mathrm{GeV}$. The Coulomb kick decreases in more peripheral collisions because the overlap volume is smaller, and therefore, less positive charge generates a smaller Coulomb field in the overlap volume.

The values of the initial pion ratios, $R_{\pi}$, decrease with increasing energy. At lower beam energies the ratios are larger than unity, which is likely due to isospin conservation and significant contributions from resonance decay (such as $\Delta$ baryons). As the energy is increasing, there is a change in pion production mechanisms and direct pion pair production dominates [14].

In our analysis, $d N^{\mathrm{ch}} / d y$ is the net-charge of the interaction region, so we subtract the antiproton $d N / d y$ from proton $d N / d y$ to get the net-proton values. The experimental values of proton and antiproton rapidity densities were taken from [14]. These net-proton $d N / d y$ values were used to obtain the kinetic freeze-out radius based on (1). The energy and centrality dependence of the kinetic freeze-out radius (solid symbols) is shown in Figure 6. The open symbols represent the chemical freeze-out radius of the system obtained by the STAR collaboration using a thermal model analysis [14]. The kinetic freeze-out radius shows no energy dependence for the energy range studied in this analysis. The same behavior was observed by the STAR collaboration for the chemical freeze-out radius. The kinetic freeze-out radius decreases from central to peripheral collisions indicating that in a central collision a larger system is formed.

For all energies, the kinetic freeze-out radius is larger than the chemical freeze-out radius in central collisions. The separation between the chemical freeze-out radius and kinetic freeze-out radius increases in more central collisions, which might suggest that the system expansion from

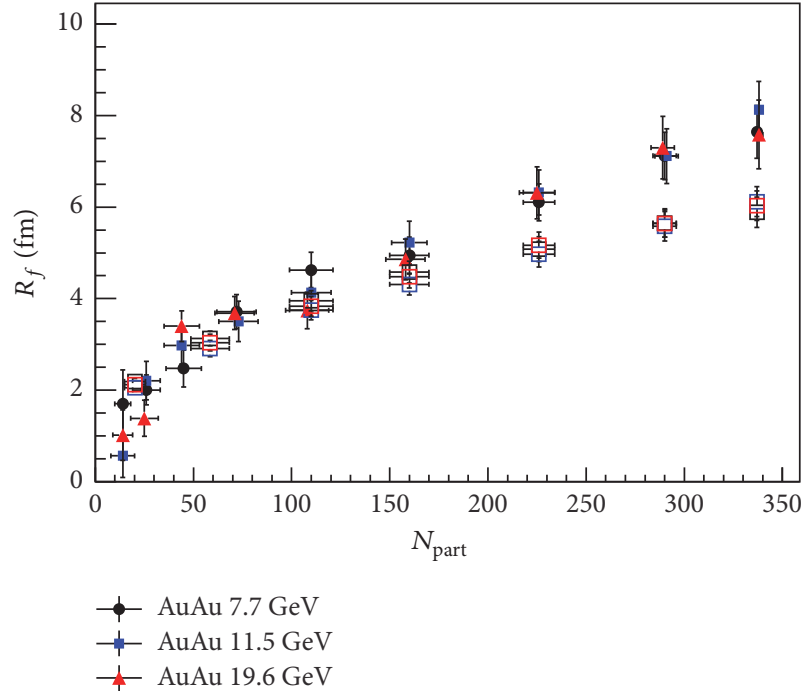

FIGURE 6: The kinetic freeze-out radius (solid symbols) as a function of collision centrality for $\mathrm{Au}+\mathrm{Au}$ collisions at $7.7 \mathrm{GeV}$ (black symbols), 11.5 GeV (blue), and 19.6 GeV (red symbols). The open symbols are the chemical freeze-out radii obtained by STAR experiment [14].

chemical freeze-out stage to thermal freeze-out is greater in these collisions.

The collision energy dependence of the Coulomb kick is shown in Figure 7. The black points are from the most central BES $\mathrm{Au}+\mathrm{Au}$ collisions, $0-5 \%$ centrality (this analysis), and the AGS $(\mathrm{Au}+\mathrm{Au}$ collisions at $11.6 \mathrm{AGeV})$ and $\mathrm{SPS}(\mathrm{Pb}+\mathrm{Pb}$ collisions at $158 \mathrm{AGeV}$ ) results were taken from [15]. To study the energy dependence of $p_{c}$, three functions $\left(f_{1}-\right.$ red curve, $f_{2}$-black dashed curve, and $f_{3}$-blue dashed curve) were also plotted on Figure 7. The used functions are

$$
\begin{aligned}
& f_{1}=a+b \ln \sqrt{s_{\mathrm{NN}}} \\
& f_{2}=a\left(\sqrt{s_{\mathrm{NN}}}\right)^{b}
\end{aligned}
$$




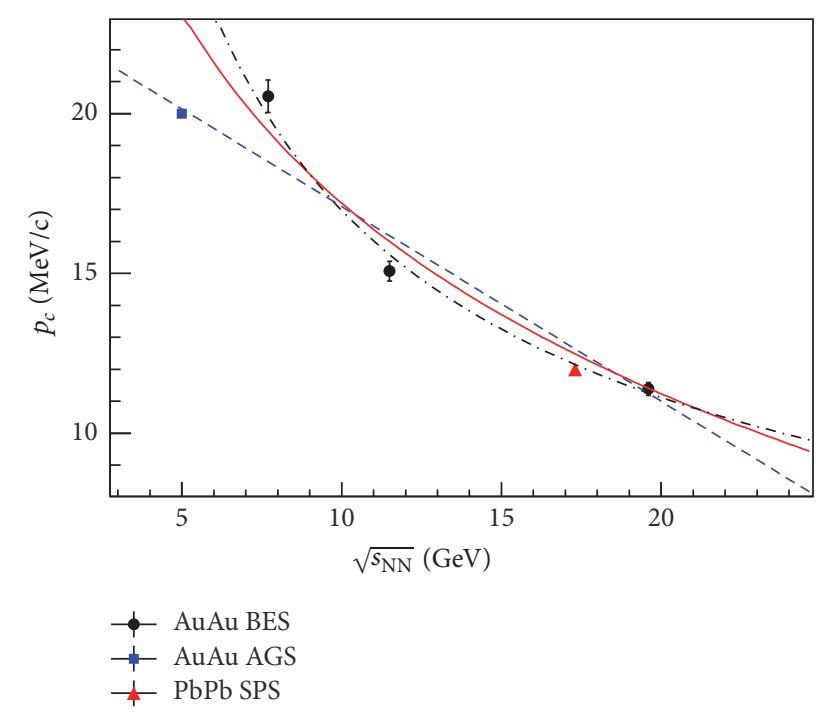

FIgURE 7: The Coulomb kick as a function of beam energy. The dashed and solid lines are the fits with three functions described in the text.

TABLE 1: The parameters of the functions used to describe the energy dependence of the Coulomb kick.

\begin{tabular}{lccc}
\hline Function & $a$ & $b$ & $\chi^{2} /$ dof \\
\hline$f_{1}$ & $36.96 \pm 1.35$ & $-8.64 \pm 0.49$ & 12.16 \\
$f_{2}$ & $69.15 \pm 5.83$ & $-0.61 \pm 0.03$ & 4.74 \\
$f_{3}$ & $23.19 \pm 0.59$ & $-0.60 \pm 0.04$ & 29.42 \\
\hline
\end{tabular}

$$
f_{3}=a+b \sqrt{s_{\mathrm{NN}}}
$$

where the values of the $a$ and $b$ parameters, as well as the $\chi^{2} /$ dof, are given in Table 1 .

The $f_{2}$ function seems to describe better the behavior of the Coulomb kick energy dependence, while the $f_{1}$ function and the linear fit fail to describe this dependence.

The Coulomb kick decreases with the increase of beam energy, showing that the Coulomb interaction is stronger at lower energies, as has been already observed in other analyses [18-20]. If the colliding nuclei are fully stopped, due to a larger stopped charge in the overlap volume a stronger Coulomb field is generated, and this Coulomb field has a stronger influence on the charged pions.

\section{Conclusions}

The results indicate stronger Coulomb effects on the $\pi^{-} / \pi^{+}$ ratio at lower beam energies. For the same collision energy, the Coulomb interaction is larger in central collisions because there is strong stopping and an important positive net-charge in the central rapidity region. The Coulomb interaction decreases in peripheral collisions. The kinetic freeze-out radius is not changing with energy for the energy interval considered in this analysis and shows an increase from peripheral to central collisions indicating the formation of a larger system in more central collisions.

\section{Conflicts of Interest}

The authors declare that there are no conflicts of interest regarding the publication of this paper.

\section{Acknowledgments}

This work was supported by IFA, RO-FAIR program, Project no. 09FAIR/16.09.2016.

\section{References}

[1] A. Wagner et al., "Evidence for different freeze-out radii of highand low-energy pions emitted in $\mathrm{Au}+\mathrm{Au}$ collisions at $1 \mathrm{~A} \cdot \mathrm{GeV}$," Physics Letters B, vol. 420, pp. 20-24, 1998.

[2] W. Reisdorf et al., "Systematics of pion emission in heavy ion collisions in the $1 \mathrm{AGeV}$ regime," Nuclear Physics A, vol. 781, pp. 459-508, 2007.

[3] L. Ahle et al., "Particle production at high baryon density in central Au+Au reactions at $11.6 \mathrm{AGeV} / \mathrm{c}$," Physical Review C, vol. 57, 466 pages, 1998.

[4] L. Ahle, "Excitation function of $\mathrm{K}+$ and $\pi+$ production in $\mathrm{Au}+\mathrm{Au}$ reactions at 2-10 AGeV," Physics Letters B, vol. 476, pp. $1-8,2000$.

[5] J. Barette et al., "Proton and pion production in $\mathrm{Au}+\mathrm{Au}$ collisions at 10.8A-GeV/c," Physical Review C, vol. 62, Article ID 024901, 2000.

[6] H. Boggild et al., "Coulomb effect in single particle distributions," Physics Letters B, vol. 372, pp. 339-342, 1996.

[7] M. Aggarwal et al., "Pion Freeze-Out Time in $\mathrm{Pb}+\mathrm{Pb}$ Collisions at $158-\mathrm{A}-\mathrm{GeV} / \mathrm{c}$ Studied via pi-/pi+ and $\mathrm{K}-/ \mathrm{K}+$ Ratios," The European Physical Journal C, vol. 48, pp. 343-352, 2006.

[8] F. Retiere et al., "Coulomb interaction of pions in central $\mathrm{Pb}+$ $\mathrm{Pb}$ collisions at 158-A-GeV," Nuclear Physics A, vol. 681, pp. 149152, 2001.

[9] W. Florkowski, "Phenomenology of ultra-relativistic heavyion collisions," Phenomenology of Ultra-Relativistic Heavy-Ion Collisions, pp. 1-416, 2010.

[10] L. Ahle et al., "Proton and deuteron production in $\mathrm{Au}+\mathrm{Au}$ reactions at $11.6 \mathrm{AGeV} / \mathrm{c}$," Physical Review C, vol. 60, Article ID 064901, 1999.

[11] H. Appelshauser et al., "Baryon stopping and charged particle distributions in central $\mathrm{Pb}+\mathrm{Pb}$ collisions at $158-\mathrm{GeV}$ per nucleon," Physical Review Letters, vol. 82, pp. 2471-2475, 1999, 2471.

[12] I. G. Bearden et al., "Nuclear stopping in $\mathrm{Au}+\mathrm{Au}$ collisions at sqrt(sNN) = 200-GeV," Physical Review Letters, vol. 93, Article ID 102301, 2004.

[13] B. Abelev et al., "Centrality dependence of $\pi, \mathrm{K}, \mathrm{p}$ production in $\mathrm{Pb}-\mathrm{Pb}$ collisions at $\mathrm{sqrt}(\mathrm{sNN})=2.76 \mathrm{TeV}$," Physical Review $C$, vol. 88, Article ID 044910, 2013.

[14] L. Adamczyk et al., "Bulk properties of the medium produced in relativistic heavy-ion collisions from the beam energy scan program," Physical Review C, vol. 96, Article ID 044904, 2017.

[15] H. W. Barz, J. P. Bondorf, J. J. Gaardhøje, and H. Heiselberg, "Coulomb effects on particle spectra in relativistic nuclear 
collisions," Physical Review C nuclear physics, vol. 57, no. 5, pp. 2536-2546, 1998.

[16] H. Heiselberg, "Freeze-out from HBT and Coulomb effects," Nuclear Physics A, vol. 638, 479C pages, 1998.

[17] K. Ackermann, "STAR detector overview," Nucl. Instrum. Meth. A, vol. 499, p. 624, 2003.

[18] D. Cebra, S. G. Brovko, C. E. Flores, B. A. Haag, and J. L. Klay, "Coulomb effect in $\mathrm{Au}+\mathrm{Au}$ and $\mathrm{Pb}+\mathrm{Pb}$ collisions as a function of collision energy," Nuclear Experiment, 6 pages, 2014.

[19] K. C. Meehan, “The fixed-target experiment at STAR," Journal of Physics: Conference Series, vol. 742, no. 1, Article ID 012022, 2016.

[20] N. Arbex, U. Ornik, M. Plümer, B. Schlei, and R. Weiner, "Pi-/pi+ ratio in heavy ion collisions: Coulomb effect or chemical equilibration?” Physics Letters B, vol. 391, no. 3-4, pp. 465-468, 1997. 

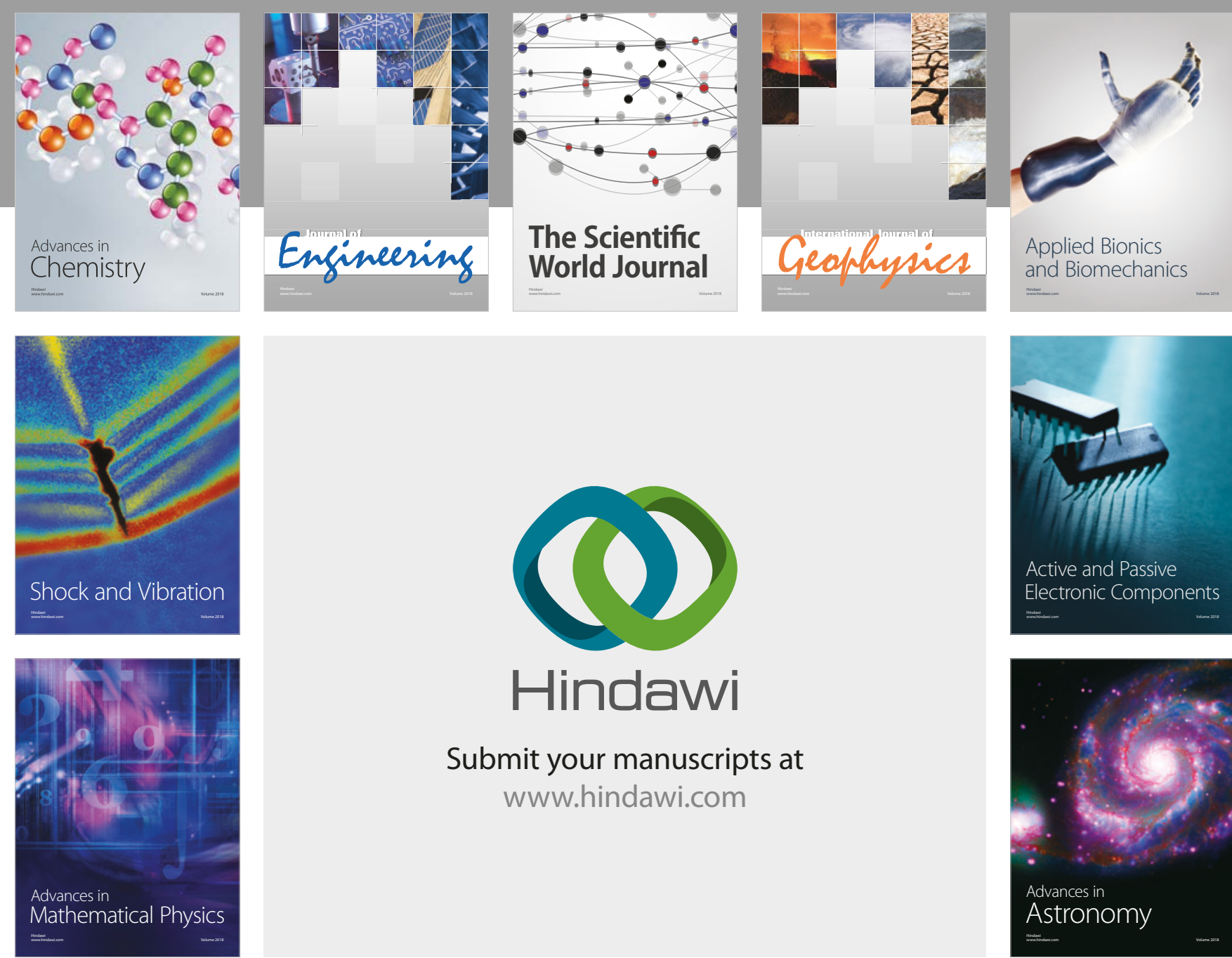

Submit your manuscripts at

www.hindawi.com

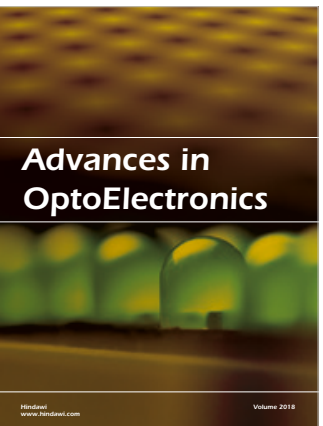

\section{Rotcting Machinery}
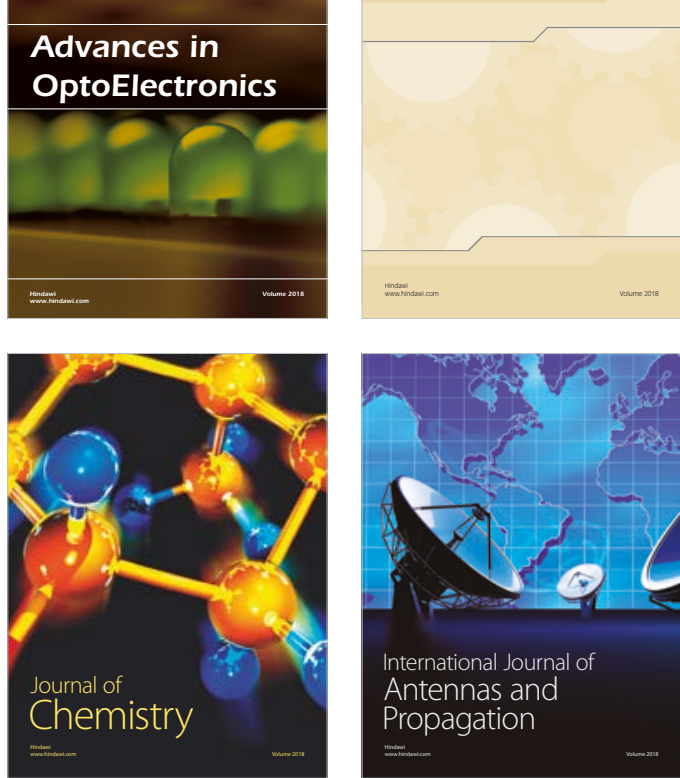

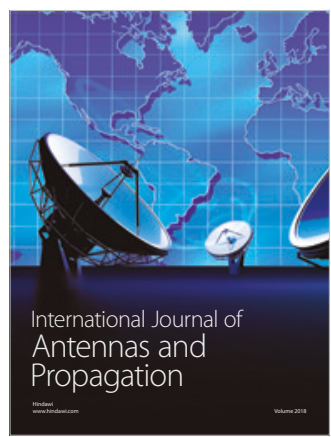

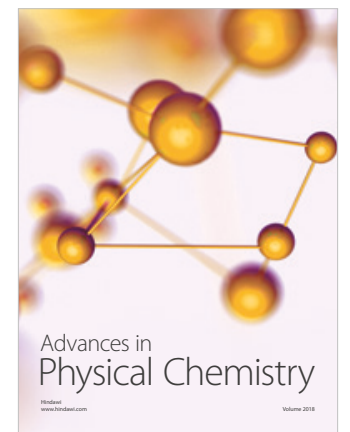

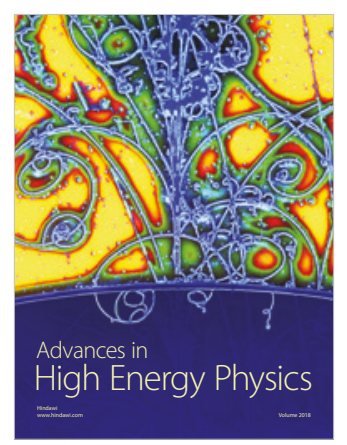

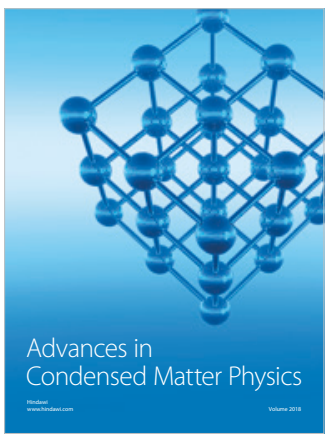

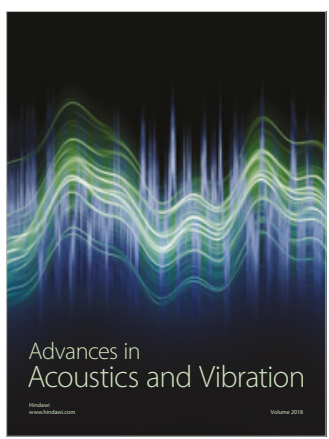

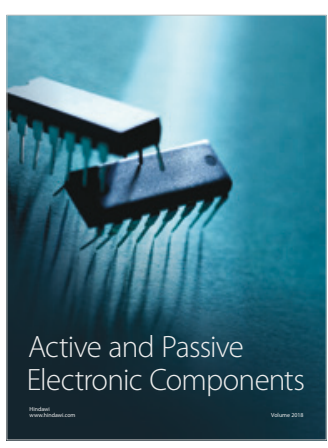
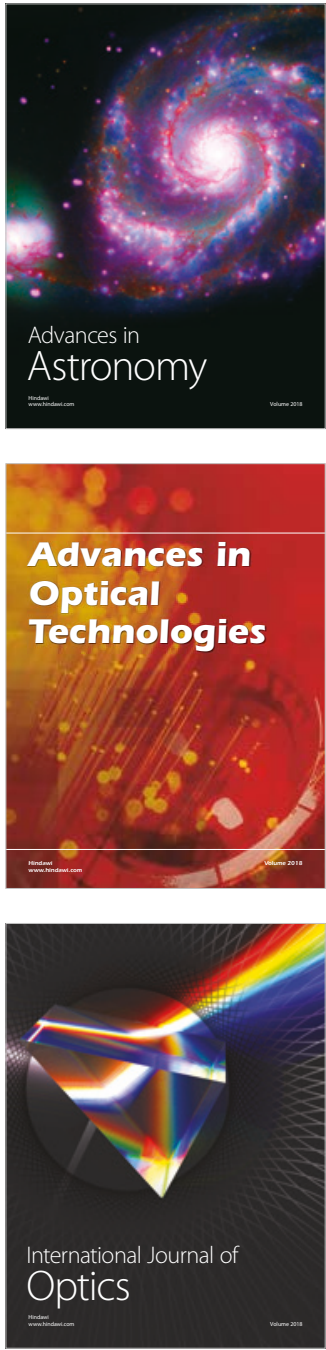